\title{
Antimicrobial resistance patterns of Uropathogens isolated from adult women with acute uncomplicated cystitis
}

\author{
Jamaan Al-Zahrani ${ }^{1}$, Khaled Al Dossari ${ }^{1}$, Ahmed H. Gabr ${ }^{2}$, Abul-fotouh Ahmed ${ }^{3}$, \\ Saad Abdulrahman Al Shahrani ${ }^{4}$ and Sameer Al-Ghamdi ${ }^{1 *}$ (D)
}

\begin{abstract}
Background: To evaluate the antibiotic resistance patterns of uropathogens isolated from adult women with acute community-acquired (CA) uncomplicated cystitis.

Results: Over a one-year period (May 2015-April 2016), the results of susceptibility testing of outpatient midstream urine samples from 5 different laboratories were prospectively evaluated. The study included only adult women with uncomplicated cystitis. The susceptibility testing in all laboratories was performed using the disk diffusion method with the VITEK-2 Compact system. The isolated uropathogens and their resistance to the tested antibiotics were evaluated. Out of 317 adult women with CA uncomplicated cystitis, 179 had a positive culture. The most commonly isolated organism was Escherichia coli (E. coli) (70.4\%), followed by Klebsiella (21.2\%). The overall resistance rate was highest for ampicillin $(85.6 \%)$, followed by cefalotin $(56.3 \%)$, trimethoprim/sulfamethoxazole (54.7\%), pipracillin (51.9\%), nitrofurantoin (48.8\%) and aztreonam (47.4\%). Isolated E. coli strains were commonly resistant to ampicillin (80.5\%), trimethoprim/sulfamethoxazole (72.2\%) and aztreonam (71.4\%), followed by cefalotin (55.9\%). The overall ciprofloxacin resistance rate was $17.9 \%$, and the resistent was found only with E. coli (25.4\%).
\end{abstract}

Conclusions: Our results may aid in the selection of proper empiric antibiotic therapy for adult women with acute CA uncomplicated cystitis.

Keywords: Antibiotic, Cystitis, Resistance, Uropathogen

\section{Background}

Urinary tract infection (UTI) is one of the most common bacterial infections seen in women. More than $50 \%$ of women develop at least one episode of UTI at some point in their lives, and many will have recurrences [1]. Although most UTIs are less severe, such infections can cause patients significant distress and are associated with high healthcare costs and social burden [2]. In adult women, acute community-acquired (CA) uncomplicated cystitis accounts for the majority of UTI and community use of antibiotics. Moreover, Escherichia coli (E. coli) is the most commonly isolated pathogen in uncomplicated

\footnotetext{
*Correspondence: sam3443@gmail.com

${ }^{1}$ Department of Family Medicine, College of Medicine, Prince Sattam bin Abdulaziz University, Al Kharj 11942, Saudi Arabia

Full list of author information is available at the end of the article
}

cystitis, accounting for $75-90 \%$ of isolates in the outpatient setting [3].

The diagnosis of acute CA uncomplicated cystitis is usually based mainly on clinical manifestations $[4,5]$. Clinicians generally prescribe empiric antibiotic therapy for uncomplicated cystitis and recommend microbial identification and susceptibility testing only in cases of recurrent UTI (rUTI), resistant infections or failure of empiric therapy $[5,6]$. The rationale for empiric antibiotic therapy is based on the predictable and narrow spectrum of the causative organisms and their susceptibility patterns. However, the misuse of antibiotics is the main origin of acquired microbial resistance to commonly prescribed antibiotics. Because of the rise in microbial resistance over time and potential differences in antibiotic susceptibility in different countries, routine assessment of antibiotic susceptibility patterns in different

(c) The Author(s). 2019 Open Access This article is distributed under the terms of the Creative Commons Attribution 4.0 International License (http://creativecommons.org/licenses/by/4.0/), which permits unrestricted use, distribution, and reproduction in any medium, provided you give appropriate credit to the original author(s) and the source, provide a link to the Creative Commons license, and indicate if changes were made. The Creative Commons Public Domain Dedication waiver (http://creativecommons.org/publicdomain/zero/1.0/) applies to the data made available in this article, unless otherwise stated. 
geographical regions is necessary to facilitate the selection of empiric therapy. The current study was conducted to determine the local antimicrobial susceptibility patterns of common uropathogens isolated from urine cultures of women with acute CA uncomplicated cystitis. The aim of the study was to identify alternative empiric antibiotic therapies that may be effective against common uropathogens in Riyadh, Saudi Arabia.

\section{Results}

Over the one-year period, a total of 2073 culture reports from outpatient urine samples were available. From those, only 317 urine samples were from adult women with CA uncomplicated cystitis. Of the 317 urine cultures, 179 (56.5\%) were positive with available sensitivity reports and were included in the data analysis.

The age of the participants ranged from 18 to 70 years (median: 28 years). The isolated uropathogen were E. coli (70.4\%), Klebsiella (21.2\%), Enterobacter (3.4\%), Proteus (2.8\%), Pseudomonas (1.7\%) and Cedecea $(0.6 \%)$. Stratified by age, the most commonly isolated uropathogen in women aged $\leq 50$ years and those $>50$ years was E. coli $(70.8 \%$ vs. $66.7 \%)$ and Klebsiella (19.9\% vs. 33.3\%). All other uropathogens, except for $E$. coli and Klebsiella, were isolated only from women $\leq 50$ years of age. No significant difference was found between both age groups for the distribution of isolated uropathogens $(P=0.685)$.

All isolated uropathogens were resistant to at least two of the tested antibiotics. Overall, the rate of antibiotic resistance was highest for ampicillin (85.6\%), cefalotin (56.3\%), trimethoprim/sulfamethoxazole (54.7\%), pipracillin (51.9\%), nitrofurantoin (48.8\%) and aztreonam (47.4\%). The overall resistance rates to ciprofloxacin and levofloxacin were 17.9 and $10.5 \%$, respectively. Among $E$. coli isolates, $80.5 \%$ were resistant to ampicillin, $72.2 \%$ to trimethoprim/sulfamethoxazole, $71.4 \%$ to aztreonam and $55.9 \%$ to cefalotin. The antibiotic susceptibility pattern for $E$. coli also demonstrated high sensitivity to nitrofurantoin, ciprofloxacin and levofloxacin. The antibiotic resistance patterns of isolated uropathogens to the tested antibiotics are shown in Table 1.

\section{Discussion}

Inappropriate use of antibiotics, excessively frequent prescription of broad-spectrum antibiotics and inadequate use of antibiotics by patients are the leading causes of bacterial gene mutations and subsequent resistance to antibiotics [7]. The study by Baddour and colleagues [8] revealed that the trend in the susceptibility pattern of methicillin-resistant Staphylococcus aureus towards antibiotics had increased to $33.8 \%$ with sulfamethoxazole/trimethoprim and $39.6 \%$ with gentamicin. Moreover, local studies demonstrated a high prevalence of multiple drug- resistant organisms and the development of extendedspectrum beta-lactamase pathogens [9].

Resistance to antimicrobial agents is considered a significant global challenge. This might be attributed to the fact that most CA-UTIs are treated without bacteriological testing. Understanding the antibiotic susceptibility patterns of causative organisms will result in a better selection of antimicrobials given as empiric therapy for UTIs.

There is a significant geographic discrepancy in in vitro susceptibility for $E$. coli. In four different studies, resistance rates were higher in Spain and Portugal than other European countries, and medical centers in the United States showed higher resistance rates than medical centers in Canada [10, 11]. The present study reports antibiotic resistance patterns of women with CA uncomplicated cystitis in our region. Most studies have identified $E$. coli as a main causative pathogen of UTI $[12,13]$. Our data confirmed that E. coli was indeed the most common causative organism of UTI (70.4\%) in our region.

The presence of Klebsiella as a causative pathogen of CA-UTI was variable among different studies. In a study from India [14] the incidence was reported to be $22 \%$, while in another study from Kuwait [15] the incidence was $10.8 \%$. In the present study, Klebsiella was the second most commonly isolated uropathogen accounting for $21.2 \%$ of cases in the studied population.

In our study, the resistance rate to ampicillin among E. coli isolates was $80.5 \%$. This is considered very high compared with previous studies that showed an ampicillin resistance rate of $30 \%$ in Canada [16] and $49 \%$ in the United Kingdom [17]. In a surveillance program, the ECO.SENSE project [18], the resistance of E. coli to ampicillin was refined according to its relation with resistance to other antimicrobials. Kahlmeter et al. [5] reported a resistance rate of $80 \%$ to ampicillin among ciprofloxacin-resistant $E$. coli strains, which is comparable to our results. For trimethoprim/sulfamethoxazole resistance, our results also indicated a much higher resistance than previous studies $(11 \%$ in Canada and $16-18 \%$ in the United States) [16]. This difference might be attributed to the ease of obtaining these antibiotics from the drug market in our country compared with tight restrictions enforced in other countries. Our finding of increased resistance to ampicillin and trimethoprim/sulfamethoxazole suggests that these agents may no longer be suitable for empiric therapy against CA-UTI. Our data showed that individual antibiotic activity was lower compared with antibiotic combinations with $\beta$-lactamase inhibitors, such as sulbactam and tazobactam, which may suggest that these bacteria may contain $\beta$-lactamase enzymes. 
Table 1 Antibiotic resistance of the isolated organisms to the tested antibiotics

\begin{tabular}{|c|c|c|c|c|c|c|c|}
\hline \multirow[t]{2}{*}{ Tested antibiotics } & \multicolumn{7}{|c|}{ Isolated organisms } \\
\hline & Overall & E.coli & Klebsiella & Enterobacter & Proteus & Pseudomonas & Cedecea \\
\hline \multicolumn{8}{|c|}{ Antibiotics tested against lactose and non-lactose fermenting organisms } \\
\hline & $(n=179)$ & $(n=126)$ & $(n=38)$ & $(n=6)$ & $(n=5)$ & $(n=3)$ & $(n=1)$ \\
\hline Amikacin & $18(10.1)$ & $18(14.3)$ & $0(0.0)$ & $0(0.0)$ & $0(0.0)$ & $0(0.0)$ & $0(0.0)$ \\
\hline Cefepime & $36(20.1)$ & $29(23.0)$ & $6(15.8)$ & $0(0.0)$ & $0(0.0)$ & $0(0.0)$ & $1(100)$ \\
\hline Ceftazidime & $36(20.1)$ & $29(23.0)$ & $6(15.8)$ & $0(0.0)$ & $0(0.0)$ & $0(0.0)$ & $1(100)$ \\
\hline Ciprofoxacin & $32(17.9)$ & $32(25.4)$ & $0(0.0)$ & $0(0.0)$ & $0(0.0)$ & $0(0.0)$ & $0(0.0)$ \\
\hline Gentamicin & $32(17.9)$ & $26(20.6)$ & $6(15.8)$ & $0(0.0)$ & $0(0.0)$ & $0(0.0)$ & $0(0.0)$ \\
\hline Imipenem & $6(3.4)$ & $0(0.0)$ & $6(15.8)$ & $0(0.0)$ & $0(0.0)$ & $0(0.0)$ & $0(0.0)$ \\
\hline Meropenem & $3(1.5)$ & $0(0.0)$ & $0(0.0)$ & $0(0.0)$ & $0(0.0)$ & $0(0.0)$ & $0(0.0)$ \\
\hline Tigecycline & $18(10.1)$ & $0(0.0)$ & $6(15.8)$ & $6(100)$ & $5(100)$ & $0(0.0)$ & $1(100)$ \\
\hline Pipracillin/Tazobactam & $25(14.0)$ & $18(14.3)$ & $6(15.8)$ & $0(0.0)$ & $0(0.0)$ & $0(0.0)$ & $1(100)$ \\
\hline Trimethoprim/Sulfamethoxazol & $98(54.7)$ & $71(72.2)$ & $6(15.8)$ & $0(0.0)$ & $0(0.0)$ & $0(0.0)$ & $1(100)$ \\
\hline \multicolumn{8}{|c|}{ Antibiotics tested against lactose fermenting organisms } \\
\hline & $(n=160)$ & $(n=118)$ & $(n=36)$ & $(n=6)$ & $(n=0)$ & $(n=0)$ & $(n=0)$ \\
\hline Amoxacillin/Clavulanic acid & $66(41.3)$ & $54(45.8)$ & $6(16.7)$ & $6(100)$ & - & - & - \\
\hline Ampicillin & $137(85.6)$ & $95(80.5)$ & $36(100)$ & $6(100)$ & - & - & - \\
\hline Cefalotin & $90(56.3)$ & $66(55.9)$ & $18(50.0)$ & $6(100)$ & - & - & - \\
\hline Cefoxitin & $48(30.0)$ & $36(30.5)$ & $6(16.7)$ & $6(100)$ & - & - & - \\
\hline Ceftriaxone & $48(30.0)$ & $36(30.5)$ & $6(16.7)$ & $6(100)$ & - & - & - \\
\hline Nitrofurantoin & $78(48.8)$ & $36(30.5)$ & $36(100)$ & $6(100)$ & - & - & - \\
\hline Pipracillin & $83(51.9)$ & $59(50.0)$ & $24(66.7)$ & $0(0.0)$ & - & - & - \\
\hline \multicolumn{8}{|c|}{ Antibiotics tested against non-lactose fermenting organisms } \\
\hline & $(n=19)$ & $(n=8)$ & $(n=2)$ & $(n=0)$ & $(n=5)$ & $(n=3)$ & $(n=1)$ \\
\hline Ampicillin/Sulbactam & $2(10.5)$ & $1(12.5)$ & $0(0.0)$ & - & $0(0.0)$ & $0(0.0)$ & $1(100)$ \\
\hline Aztreonam & $9(47.4)$ & $5(62.5)$ & $0(0.0)$ & - & $0(0.0)$ & $3(100)$ & $1(100)$ \\
\hline Colistin & $8(42.1)$ & $0(0.0)$ & $0(0.0)$ & - & $5(100)$ & $3(100)$ & $0(0.0)$ \\
\hline Ertapenem & $3(15.8)$ & $2(25.0)$ & $0(0.0)$ & - & $0(0.0)$ & $1(0.0)$ & $1(100)$ \\
\hline Levofloxacin & $2(10.5)$ & $2(25.0)$ & $0(0.0)$ & - & $0(0.0)$ & $0(0.0)$ & $0(0.0)$ \\
\hline Minocycline & $8(42.1)$ & $2(25.0)$ & $0(0.0)$ & - & $5(100)$ & $0(0.0)$ & $1(100)$ \\
\hline Ticarcillin/Clavulanic acid & $8(42.1)$ & $2(25.0)$ & $0(0.0)$ & - & $5(100)$ & $0(0.0)$ & $1(100)$ \\
\hline
\end{tabular}

Data presented as number (percentage)

Fluoroquinolones have been suggested as reasonable empiric treatment for CA-UTI [13]. With a resistance rate of $17.9 \%$ for ciprofloxacin and $10.5 \%$ for levofloxacin in our study, this could be plausible. However, the increasing use of this antimicrobial group raises concerns regarding the possibility of increasing resistance patterns in the near future. Our concerns are further validated by the recent international clinical practice guidelines, which recommended fluoroquinolones as an alternative agent for the treatment of uncomplicated UTIs, rather than the previously recommended first-line therapy [19]. Additionally, this is in agreement with the recent announcement of the US Food and Drug Administration. The package insert of fluoroquinolones will be updated to include a warning stating that this antimicrobial agent should not be used for routine respiratory tract infections or uncomplicated UTIs unless there is no suitable alternative agent [20].

Isolated E. coli strains were found to be the most susceptible to imipenem, meropenem, tigecycline and colistin. The apparent lack of resistance to these antibiotics may be explained by its limited availability in our region and its high cost.

There are some limitations associated with this study. First, the study population was limited to one locality. A larger cohort of patients from different geographical regions with different health conditions with study of the mechanisms through which bacteria develop resistance 
may provide more informative data. Second, the results of our study were based on culture reports from five different laboratories with different laboratory procedures, which might have led to variations in resistance rates. Finally, possible side effects associated with each antimicrobial agent were not reported. However, such side effects were not anticipated to affect the rates of antimicrobial resistance.

\section{Conclusions}

E. coli remains the most commonly isolated Gramnegative causative organism of UTI, followed by Klebsiella. Isolated uropathogens demonstrated high sensitivity to imipenem, meropenem, Ampicillin/Sulbactam and levofloxacin. As an oral and cost-effective therapy, we recommend that levofloxacin be used as alternative antimicrobial treatment for CA uncomplicated cystitis. Further studies with a larger number of patients in different regions should be performed to recommend national guidelines for the treatment of CA-UTI.

\section{Methods}

Data were collected from 5 clinical microbiology laboratories and in the province of Riyadh, Saudi Arabia, during the one-year period between May 2015 and April 2016. All patients were referred to the laboratories either by Urologists or primary health care physicians at two University hospitals and three primary health care centers. Patients' medical files were reviewed to limit the analysis to CA uncomplicated cystitis. Adult women (at least 18 years of age) who presented at outpatient clinics with symptoms of acute cystitis (dysuria, urgency, frequency, hematuria, or suprapubic pain) and agreed to participate in the study were prospectively recruited. Women with a history of rUTI (defined as three or more UTI episodes in the past year or two or more episodes in the past 6 months), recent antibiotic treatment within the past two weeks, immunocompromised state, pregnancy, urinary catheter, associated urinary tract pathology or anatomical abnormalities and those with history of hospitalization within the past month were excluded. Ethics approval for the study project was obtained from the Deanship of Scientific Research at Prince Sattam Bin Abdulaziz University. All study procedures were performed in accordance with the principles laid down in the Helsinki Declaration (1975) and its later amendments.

A midstream urine sample was collected by each participant in a sterile container and sent to the main laboratory within $20 \mathrm{~min}$ for culture and sensitivity analysis and processed within $2 \mathrm{~h}$. Cultures that showed atypical organisms and mixed organisms suggestive of contamination were excluded from the analysis. A urine culture was considered to be positive for infection if the colony count for a single organism was more than $10^{5}$ colony-forming units/ml.

All isolates were identified and tested for susceptibility by the Vitek 2 system (bioMérieux; Inc., USA) using the Gram-negative strain cards AST-N291 and AST-P580. The isolates were tested for susceptibility for the following antimicrobial agents: amikacin, cefepime, ceftazidime, ciprofloxacin, gentamycin, imipenem, meropenem, tigecycline, pipracillin/tazobactam, trimethoprim/sulfamethoxazole, amoxicillin/clavulanate, ampicillin, cefalotin, cefoxitin, ceftriaxone, nitrofurantoin, pipracillin, ampicillin/sulbactam, aztreonam, colistin, ertapenem, levofloxacin, minocycline, ticarcillin/clavulanic acid. The cards were inoculated and incubated in the system according to the manufacturer's instructions. All results were interpreted using the Advanced Expert System (software version VT2-R04.03). Using the Vitek 2 system AST-N0291 card, the isolates were initially considered positive for extended spectrum beta-lactemase (ESBL) if the minimum inhibitory concentration of ceftazidime and cefotaxime for these organisms was $\geq 2 \mathrm{mg} / \mathrm{L}$.

The proportions of overall resistance and individual resistance of isolated uropathogens were calculated for each of the tested antibiotics. Differences in the distribution of isolates by age groups were determined using the chi-square test or Fisher's exact test. Differences were considered significant at $P$ values of $<0.05$. Statistical analyses were performed using SPSS version 25 (SPSS Inc., Chicago, IL).

\section{Abbreviations \\ CA: Community-acquired; E. coli: Escherichia coli; rUTI: Recurrent urinary tract infection; UTI: Urinary tract infection}

\section{Acknowledgments}

We thank Dr. Shafqat Ahmed in the microbiology laboratory at Prince Sattam bin Abdulaziz University Hospital for his assistance.

\section{Authors' contributions}

This work was performed as a collaboration among all of the authors. JAZ participated in the study design and wrote the first draft of the manuscript. KAD and AHG collected and processed the samples. AAA, SAA and SAG participated in the study design and performed the statistical analyses. All of the authors read and approved the final manuscript.

\section{Funding}

Deanship of Scientific Research at Prince Sattam Bin Abdulaziz University under research project \#2015/03/4768. Funding was in term of financial support of the work team and the provision of equipment and tools in addition to ensuring the costs of transportation and communications.

\section{Availability of data and materials}

Data are available upon request from the authors.

\section{Ethics approval and consent to participate}

The study was approved by Deanship of Scientific Research at Prince Sattam bin Abdulaziz University under research project \#2015/03/4768.. Letters of authorization were obtained from Prince Sattam bin Abdulaziz University Hospital and Riyadh Care Hospital prior to data collection and written informed consent was obtained from the participants. 


\section{Consent for publication}

Not applicable.

\section{Competing interests}

The authors declare that they have no competing interests.

\section{Author details}

'Department of Family Medicine, College of Medicine, Prince Sattam bin Abdulaziz University, Al Kharj 11942, Saudi Arabia. ${ }^{2}$ Department of Urology, Minia University, Minia, Egypt. ${ }^{3}$ Department of Urology, Al-Azhar University, Cairo, Egypt. ${ }^{4}$ Department of Urology, College of Medicine, Prince Sattam bin Abdulaziz University, Al Kharj 11942, Saudi Arabia.

Received: 3 September 2018 Accepted: 15 October 2019

Published online: 30 October 2019

\section{References}

1. Hooton TM. Clinical practice. Uncomplicated urinary tract infection. N Engl J Med. 2012;366(11):1028-37.

2. Foxman B. Urinary tract infection syndromes: occurrence, recurrence, bacteriology, risk factors, and disease burden. Infect Dis Clin N Am. 2014; 28(1):1-13.

3. Suskind AM, Saigal CS, Hanley JM, Lai J, Setodji CM, Clemens JQ. Incidence and Management of Uncomplicated Recurrent Urinary Tract Infections in a National Sample of women in the United States. Urology. 2016;90:50-5.

4. Sheerin NS. Urinary tract infection. Medicine. 2011;39(7):384-9.

5. Kahlmeter G, Poulsen HO. Antimicrobial susceptibility of Escherichia coli from community-acquired urinary tract infections in Europe: the ECO.SENS study revisited. Int J Antimicrob Agents. 2012;39(1):45-51.

6. Alzohairy $\mathrm{M}$, Khadri $\mathrm{H}$. Frequency and antibiotic susceptibility pattern of uro-pathogens isolated from community and hospital-acquired infections in Saudi Arabia-a prospective case study. Brit J Med Medical Res. 2011;1(2):45

7. Mellon M, Benbrook C, BENBROOK L. Estimates of antimicrobial Abusein livestock, vol. 9. Washington: Union of Concerned Scientists Publications; 2001.

8. Baddour MM, Abuelkheir MM, Fatani AJ. Trends in antibiotic susceptibility patterns and epidemiology of MRSA isolates from several hospitals in Riyadh, Saudi Arabia. Ann Clin Microbiol Antimicrob. 2006;5:30.

9. Ahmad S, Al-Juaid NF, Alenzi FQ, Mattar EH, Bakheet OS. Prevalence, antibiotic susceptibility pattern and production of extended-spectrum betalactamases amongst clinical isolates of Klebsiella pneumoniae at armed forces Hospital in Saudi Arabia. J Coll Physicians Surg Pak. 2009;19(4):264-5.

10. Kahlmeter G. Prevalence and antimicrobial susceptibility of pathogens in uncomplicated cystitis in Europe. The ECO. SENS study. Int J Antimicrob Agents. 2003;22:49-52.

11. Zhanel GG, Hisanaga TL, Laing NM, DeCorby MR, Nichol KA, Weshnoweski B, et al. Antibiotic resistance in Escherichia coli outpatient urinary isolates: final results from the north American urinary tract infection collaborative Alliance (NAUTICA). Int J Antimicrob Agents. 2006;27(6):468-75.

12. Gupta K, Hooton TM, Stamm WE. Increasing antimicrobial resistance and the management of uncomplicated community-acquired urinary tract infections. Ann Intern Med. 2001;135(1):41-50.

13. Karlowsky JA, Kelly LJ, Thornsberry C, Jones ME, Sahm DF. Trends in antimicrobial resistance among urinary tract infection isolates of Escherichia coli from female outpatients in the United States. Antimicrob Agents Chemother. 2002;46(8):2540-5.

14. Akram M, Shahid M, Khan AU. Etiology and antibiotic resistance patterns of community-acquired urinary tract infections in JNMC hospital Aligarh, India. Ann Clin Microbiol Antimicrob. 2007:6(1):1

15. Al Benwan K, Al Sweih N, Rotimi VO. Etiology and antibiotic susceptibility patterns of community-and hospital-acquired urinary tract infections in a general hospital in Kuwait. Med Princ Pract. 2010;19(6):440-6.

16. Mclsaac WJ, Mazzulli T, Moineddin R, Raboud J, Ross S. Uropathogen antibiotic resistance in adult women presenting to family physicians with acute uncomplicated cystitis. Can J Infect Dis Medical Microbiol. 2004;15(5):266

17. Farrell D, Morrissey I, De Rubeis D, Robbins M, Felmingham D. A UK multicentre study of the antimicrobial susceptibility of bacterial pathogens causing urinary tract infection. J Infect. 2003:46(2):94-100.

18. Kahlmeter $\mathrm{G}$. An international survey of the antimicrobial susceptibility of pathogens from uncomplicated urinary tract infections: the ECO.SENS project. J Antimicrob Chemother. 2003;51(1):69-76.
19. Gupta K, Hooton TM, Naber KG, Wullt B, Colgan R, Miller LG, et al. International clinical practice guidelines for the treatment of acute uncomplicated cystitis and pyelonephritis in women: a 2010 update by the Infectious Diseases Society of America and the European Society for Microbiology and Infectious Diseases. Clin Infect Dis. 2011;52(5):e103-e20.

20. US Food and Drug Administration. Fluoroquinolone antibacterial drugs. Drug Safety Communication - FDA advises restricting use for certain uncomplicated infections. Posted May12,2016.http://www.fda.gov/Safety/ MedWatch/SafetyInformation/SafetyAlertsforHumanMedicalProducts/ucm5 00665.htmAccessed May 15, 201 .Accessed May 15, 2016.

\section{Publisher's Note}

Springer Nature remains neutral with regard to jurisdictional claims in published maps and institutional affiliations.
Ready to submit your research? Choose BMC and benefit from:

- fast, convenient online submission

- thorough peer review by experienced researchers in your field

- rapid publication on acceptance

- support for research data, including large and complex data types

- gold Open Access which fosters wider collaboration and increased citations

- maximum visibility for your research: over $100 \mathrm{M}$ website views per year

At BMC, research is always in progress.

Learn more biomedcentral.com/submissions 